\title{
Towards Anthropomorphic Movements for Industrial Robots
}

\author{
Christian Brecher ${ }^{1}$, Simon Müller ${ }^{1, *}$, Sinem Kuz ${ }^{2}$, and Wolfram Lohse ${ }^{1}$ \\ ${ }^{1}$ Laboratory for Machine Tools and Production Engineering WZL, RWTH Aachen University \\ \{c.brecher, s.mueller,w. lohse\}@wzl.rwth-aachen. de \\ ${ }^{2}$ Institute of Industrial Engineering and Ergonomics, RWTH Aachen University \\ s.kuz@iaw.rwth-aachen.de
}

\begin{abstract}
In order to increase productivity for processes that involve the interaction of human and robot, a promising approach is to increase the transparency of robot movements. Based on the hypothesis that anthropomorphic movements are more transparent to a human operator, this paper presents methodologies and techniques to generate humanlike movements for industrial robots.
\end{abstract}

Keywords: Anthropomorphic movements, industrial robot, movement parameters.

\section{Introduction}

To meet the increasing challenges in regard to production in high-wage countries, one of the approaches has been to automate assembly tasks using industrial robots. In today's production plants - in an increasing number of scenarios - these robots interact with human operators in cooperative environments. As the requirements regarding productivity increase, the naïve approach is to increase automation as well. According to the "law of diminishing returns", however, an increase in automation will likely not lead to a significant increase in productivity but can also have adverse effects. According to Kinkel the amount of process errors is in average significantly reduced by automation, but the severity of potential consequences of a single error increases disproportionately [1]. These "Ironies of Automation" which were identified by Lisanne Bainbridge can be considered as a vicious circle, where a function that was allocated to a human operator due to poor human reliability is automated [2]. Thus the automation results in higher function complexity, finally increasing the demands on the human operator for planning, teaching and monitoring, and hence leading to a more error-prone system.

In order to break cited vicious circle for assembly processes that involve the interaction of human and robot, a promising approach is to change the movement of the industrial robot, such that it resembles a human movement (anthropomorphic movements). This hypothesis is based on the findings by Kuz et al. towards the anticipation of a robot's movement characteristics by a human operator [3]. In the studies a virtual environment for the assembly in a predetermined working space was 
setup and the expectation of the human operator was compared to the actual behavior of the robot. The foundations for the behavior of the virtual robot were movement trajectories of a human assembly operation that was acquired by a motion capture system. Due to the fact that it was a virtual scenario, the robot's control unit was able to control the robot in at every single point in time in all of the degrees of freedom.

The robot control unit of an industrial robot, however, provides only a limited set of predefined movement types, e.g. Point-To-Point (PTP) or Linear (LIN). In a fully automated scenario these types suffice to reach every point in the workspace of a robot from a predefined direction. The focus of these commands is on a specific objective like a certain speed or the shape of the path. The opportunities to vary the set of parameters for both speed and path are very limited. A human movement, however, is defined by a complex pattern for both speed and path throughout the motion from one point to another.

This paper presents methodology and techniques to transform human movement trajectories so that they can be executed by industrial robots. Chapter 2 describes the advantages of this methodology and the challenges of its application for industrial robots. The main model for the methodology is described in Chapter 3, followed by an outline of the application to a near-reality production environment in Chapter 4. The paper finishes with a description of first experimental results in Chapter 5 and concluding remarks in Chapter 6.

\section{$2 \quad$ Human and Robot Movements}

\subsection{Anthropomorphic Movements}

Research of the last years indicates that by transferring anthropomorphic features such as appearance to non-human entities, a higher user acceptance can be achieved [4-5]. Hence, especially research in the field of human-robot cooperation focusses increasingly on the design of robots with anthropomorphic characteristics to enable teamwork between man and machine. However the adaptation of human-like characteristics to an industrial robot is rarely investigated because efficiency and robustness are the most important aspects in industrial environments. Nevertheless, direct interaction between humans and robotic systems in industrial environments is a promising approach to combine the specific skills of the human and the robotic system and to increase the flexibility of the production system. Besides the aspect of physical safety, the level of trust of the human and the transparency of the system behavior are important factors for a successful collaboration. Hence, especially concerning complex automation systems, it might occur that the system behavior cannot always be anticipated by the human operator. Therefore, from a user centered point of view an important basic requirement for effective human-robot cooperation is to achieve conformity with operator expectations so that she/he can always understand the actions and intentions of the corresponding system. Thus, the advantages of anthropomorphism could also be applied for cooperative work of humans and robots in industrial environments. Accordingly, the question is whether augmenting industrial robot with anthropomorphic features would achieve a better anticipation of the robot's behavior by the human worker. 


\subsection{Robot Movement Commands}

Most of the research in regard to anthropomorphic robots has been evaluated in scenarios involving custom made robots that provide open control structures. Industrial robot control units, however, usually offer only a limited set of predefined movement types, e.g. Point-To-Point (PTP) or Linear (LIN) (cp. Figure 1, left). The PTP command provides the fastest movement between two points but the trajectory is usually not a straight line between them. In a LIN movement, the trajectory is the straight-lined path between starting and target positions. For each of these movement types robot control units offer the possibility to set both the maximum velocity and maximum acceleration. If a movement is comprised of more than two points smoothing can be used to increase the overall speed for a movement (cp. Figure 1, right). If smoothing is enabled the robot does not need to reach the exact intermediate point but just needs to enter the smoothing area that is defined by the intermediate point and a maximum smoothing distance. In order to supply an industrial robot with the capability to move humanlike, the predefined movement types have to be utilized and parameterized to form a new movement command that resembles the pattern of a human movement.
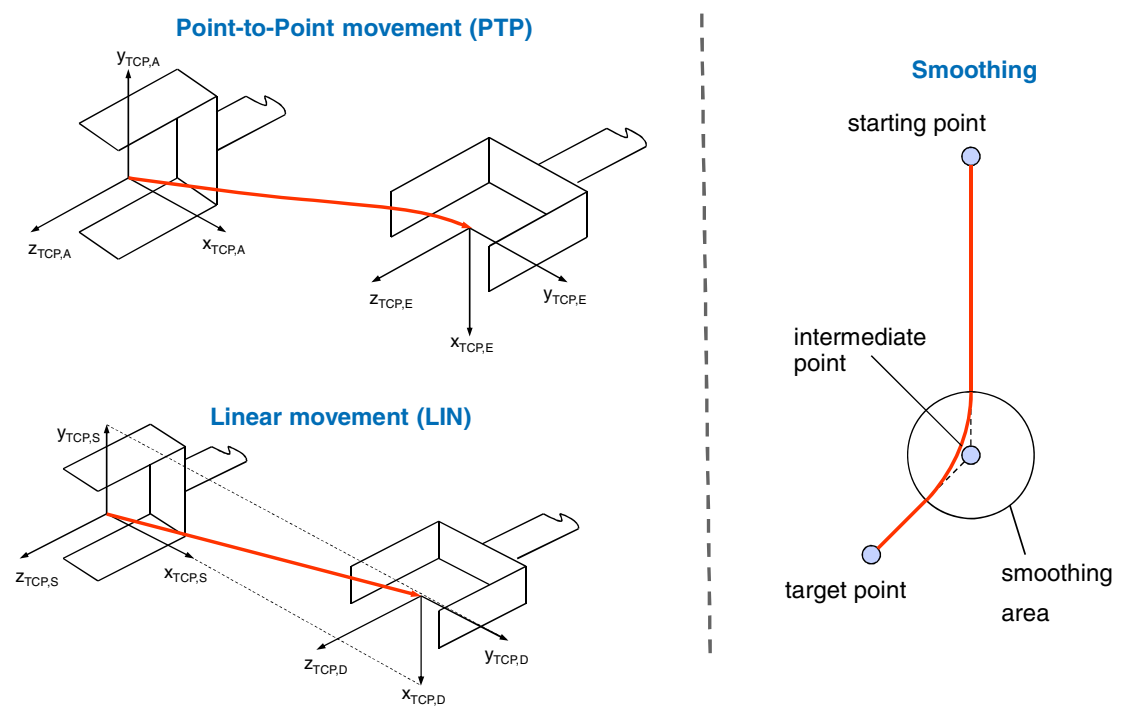

Fig. 1. Trajectories of robot movement commands PTP and LIN (left) and Smoothing (right)

\section{Framework for Generating Human Like Movements}

Figure 2 presents an overview of the framework that is used to create functions for anthropomorphic robot movement commands. In this context the term framework refers to a combination of methodologies and techniques as well as their concrete implementation in a technical system - hardware and software. The approach consists of three parts: 
1. Human tracking and trajectory extraction

2. Robot movement generation and tracking

3. Robot trajectory optimization

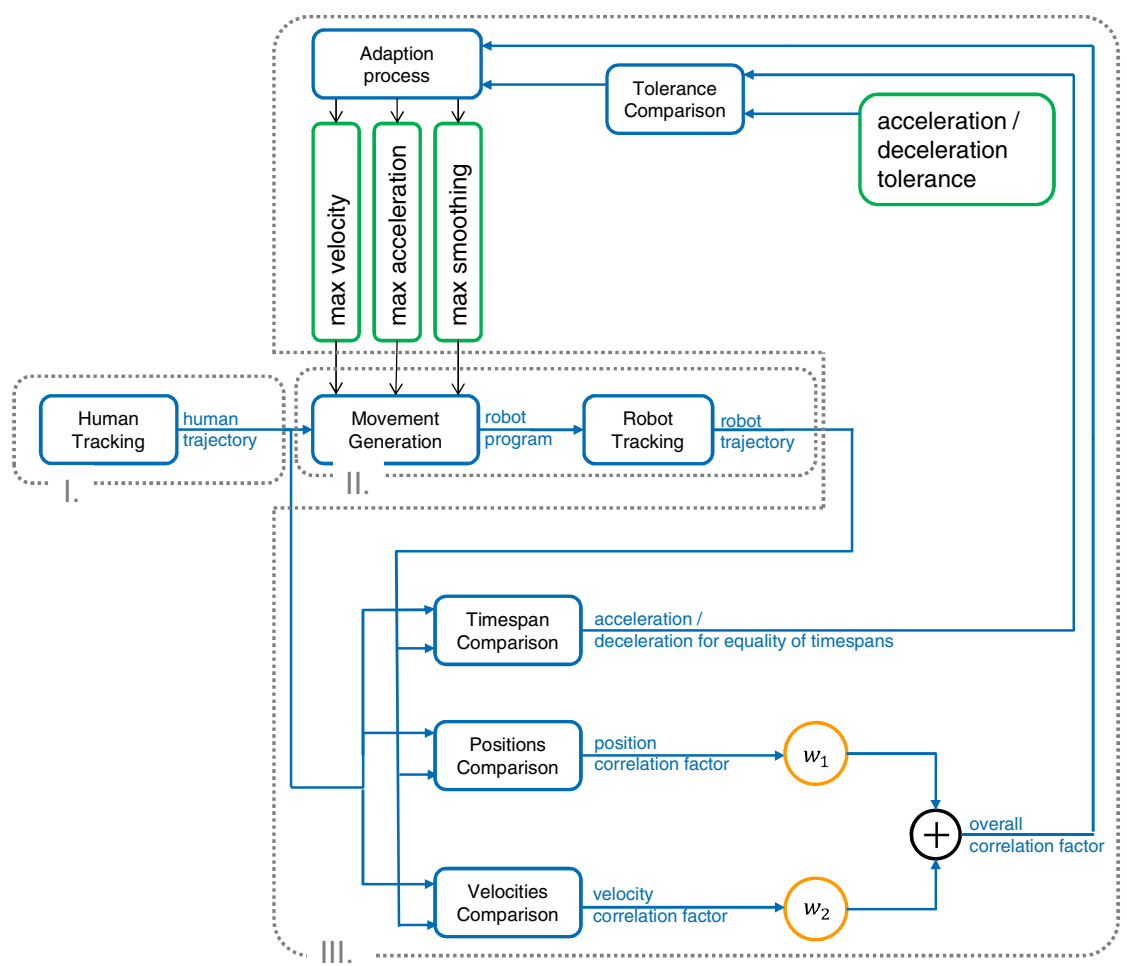

Fig. 2. Overview of framework to create anthropomorphic robot movements

\subsection{Human Tracking and Trajectory Extraction}

The first part deals with the requirement to acquire a technical representation of the human movement. In order apply anthropomorphic movement operations to an industrial robot, the trajectory of the human hand during the movement needs to be recorded. In regard to hardware, 3D markers and an optical motion capture system are utilized to record the position of the human wrist during the execution of the desired movement operation. The system captures hand trajectories as well as the duration to complete the operation. The human movement trajectories recorded by the motion tracking system - i.e. the $\mathrm{x}, \mathrm{y}$ and $\mathrm{z}$ coordinates of the end-effector at every sampling instance - are stored in an XML file. Based on this information, the human movements are analyzed and information on speed and shape of the path are extracted. 


\subsection{Robot Movement Generation and Tracking}

To find the set of movement commands that create an anthropomorphic movement for an industrial robot, different movement types have to be evaluated and the parameters for the movement types have to be varied. These trajectories form the basis for the robot movement.

The process of creating a robot program from the movement data has been automated using MATLAB. After importing a trajectory, the basic robot program structure is extended by the commands needed to enable the robot to perform the movements. Generating the robot program in MATLAB rather than by hand has many advantages, e.g. that corrections of motion tracking system measurement errors as well as adaptations of the number of data points along the trajectory can be accomplished automatically.

\subsection{Robot Trajectory Optimization}

In order to generate a robotic movement that resembles the human movement, the set of parameters for the robotic movement need to be varied. There are three parameters that can be modified: Maximum velocity, maximum acceleration and maximum smoothing (cp. Chapter 2). Due to the restrictions in maximum speed, acceleration and smoothing of the robotic movement, the robot is not always able to perform movements in the same timeframe as a human. Since industrial robots are usually used to perform value-adding activities, this implies a potential reduction of productivity. As described in the second chapter, this reduction might be compensated by an optimized interaction with a human operator. However, since studies have shown that a reduction in speed might lead to a false interpretation of movement information, a tolerance for the reduction in speed needs to be set [6]. In order to adapt the parameters for the robotic movement an optimization function is defined utilizing the correlation factor between the positions $\left(\rho_{\text {pos }}\right)$ and velocities $\left(\rho_{\text {vel }}\right)$ of the human movement and the robotic movement, respectively. For both correlation factors, the signals of the human movement is stretched in time to match the movement of the robot so that a correlation factor can be calculated. The optimization function thus results as:

$$
\begin{gathered}
\rho^{*}=\arg \max _{\{v \in \mathbb{R} \mid 0 \leq v \leq 1\},\{a \in \mathbb{R} \mid 0 \leq a \leq 1\},\{s \in \mathbb{N} \mid 0 \leq s \leq 100\}}\left(\rho_{\text {overall }}(v, a, s)\right) \\
=\arg \max _{\{v \in \mathbb{R} \mid 0 \leq v \leq 1\},\{a \in \mathbb{R} \mid 0 \leq a \leq 1\},\{s \in \mathbb{N} \mid 0 \leq s \leq 100\}}\left(w_{1} * \rho_{\text {pos }}(v, a, s)+w_{2} * \rho_{\text {vel }}(v, a, s)\right), \\
w_{1}+w_{2}=1,\left\{w_{1} \in \mathbb{R} \mid 0 \leq w_{1} \leq 1\right\},\left\{w_{2} \in \mathbb{R} \mid 0 \leq w_{2} \leq 1\right\}
\end{gathered}
$$

The weighting factors $w_{1}$ and $w_{2}$ express a human's capability to recognize positions and velocities of movements. They are necessary due to the inherent conflict of objectives for achieving the exact positions and velocities as the human. For example, without smoothing enabled the trajectory of the robot is almost exactly the same as the humans. In regard to speed, however, there is little to no correlation between the two movements, since the robot stops at every intermediate point of the trajectory. 
In the adaptation process the optimization is performed for those parameter combinations that fulfill the requirements regarding the timespan tolerance of the robotic movement.

The result of this model's application are functions for the robot control unit that provide the robot with a movement that is most similar to that of a human for a given operation. These functions can be utilized to further investigate on the perception of robotic movements from an operator's point of view. Thus, the framework comprises the basis for further laboratory tests in a near-reality production environment on a human operator's perception of anthropomorphic movements for industrial robots. Furthermore, the framework could be used to improve aspects of programming industrial robots e.g. in Programming-by-Demonstration based solutions [7].

\section{Evaluation Scenario}

\subsection{Movement Recordings}

In order to make use of the designed system in a production system, the evaluation is based on common pick and place movements. For the recording and tracking of the movements an infrared optical tracking system has been used in a scenario where 20 fields comprise a grid of possible target positions (cp. Figure 3). In a previous study the position data for all fields has been recorded, analyzed and transformed into a virtual scenario (see [3]). The gathered information is utilized for the generation of robot programs as well as the comparison in following steps. Since the target positions in the virtual scenario have a distance ranging between about $50 \mathrm{~mm}$ and $400 \mathrm{~mm}$ from the starting positions, they give a valid representation of real life pick and place operations in an industrial environment.

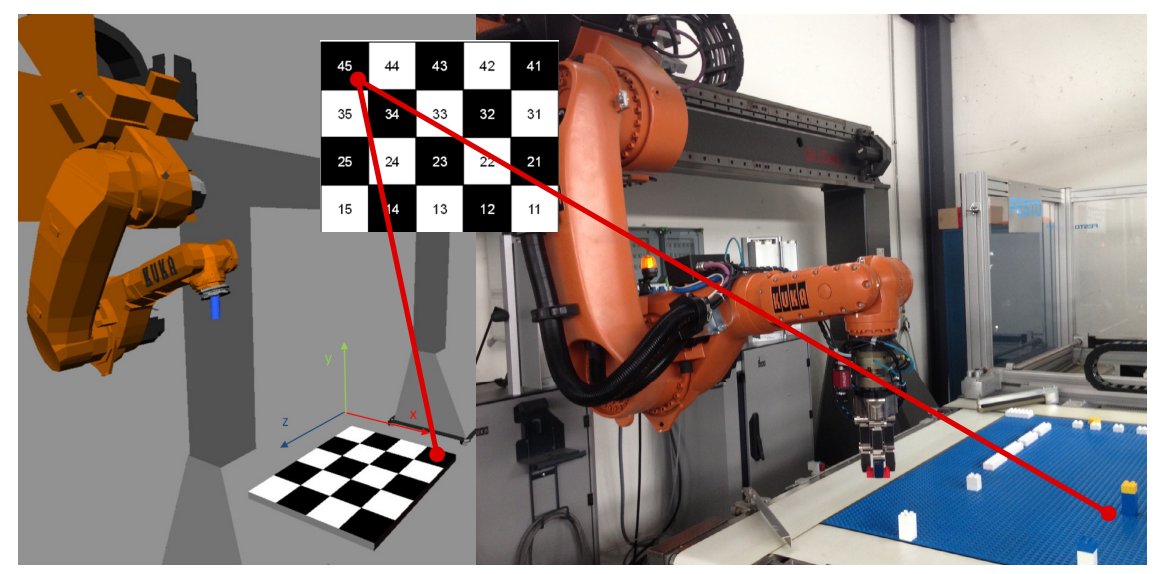

Fig. 3. Evaluation scenario in virtuality (left) and reality (right) 


\subsection{Robot Cell}

To evaluate the designed framework in a near-reality production environment a validation scenario comprised of an industrial robot (KUKA KR 30 Jet) and a dexterous hand (SCHUNK Dexterous Hand 2) has been set up. This scenario is controlled by a system that is designed like the described framework and thus generates robot control programs utilizing different human movement patterns. When these programs are executed on the robot control unit, the system analyzes their resemblance to the human movement and adjusts the parameters of the used movement types according to possible differences.

Generally, robot control units such as the KUKA Robot Control (KRC) have only limited means to communicate with external systems, e.g. through digital or analogue inputs and outputs. This originates from their common use in highly automated environments where they perform predefined tasks. However, to analyze the robot movement trajectories, a link to an external PC is necessary.

For KUKA robots the Robot Sensor Interface (RSI) is an extension to the basic KUKA Robot Language (KRL), which offers the possibility to process sensor inputs in real-time, working with interlinked signal processing blocks. Moreover, it offers the means for communication with an external system. An XML string that contains information such as the tool center point position, orientation and the axis values is sent via TCP/IP from the KRC to an externals system every $12 \mathrm{~ms}$. The external system has to reply with an XML string of predefined structure within $4 \mathrm{~ms}$ of reception. Hence, these XML strings can be used to send variable values in both directions.

A MATLAB interface for this XML communication has been designed that satisfies the used protocol and real-time constraints. Hereby it is possible to record the robot movement trajectories which are later compared to that of human movements. Moreover, it is possible to vary robot movement parameters like the velocity and smoothing during program execution, without having to edit and reload the robot program.

This interface is used to initiate movement sequences defined in the robot program and record the movement trajectory. While the interface also offers the possibility of controlling the TCP position and orientation from MATLAB, this is not desirable in our case.

\subsection{Parameter Adaptation}

In order to use the methodology from chapter 3 , the variables $w_{1}$ and $w_{2}$ as well as the allowed acceleration need to be set. Since the focus of this paper is on the technical aspects of anthropomorphic robot movements, $w_{1}$ and $w_{2}$ are arbitrarily fixed to 0.5 . In future, these values need to be determined by experimental studies. In addition to the two objectives - position and velocity - other potential objectives might result from these experimental studies, thus also increasing the number of weighting factors. For the acceleration tolerance no restrictions have been made in this scenario. 


\section{Experimental Results}

Figure 4 presents an exemplary result for a robotic movement in PTP mode with a maximum acceleration of $80 \%$, maximum velocity of $20 \%$ and a maximum smoothing of $20 \mathrm{~mm}$ to the first row and first column grid in the scenario. The charts on the left hand side present the $\mathrm{x}, \mathrm{y}$ and $\mathrm{z}$ coordinates of the robot's end effector and the human wrist over time, while the right hand side presents the velocities in $\mathrm{x}, \mathrm{y}$ and $\mathrm{z}$ direction of the shared coordinate system. The execution time for the movements has already been adapted so that the correlation factors can be calculated. Given that the weighting factor $w_{1}$ and $w_{2}$ are set to 0.5 the chosen set of parameters achieves a high overall correlation factor $-\rho_{\text {overall }}(0.8,0.2,20) \approx 0.9171-$ for this movement operation.
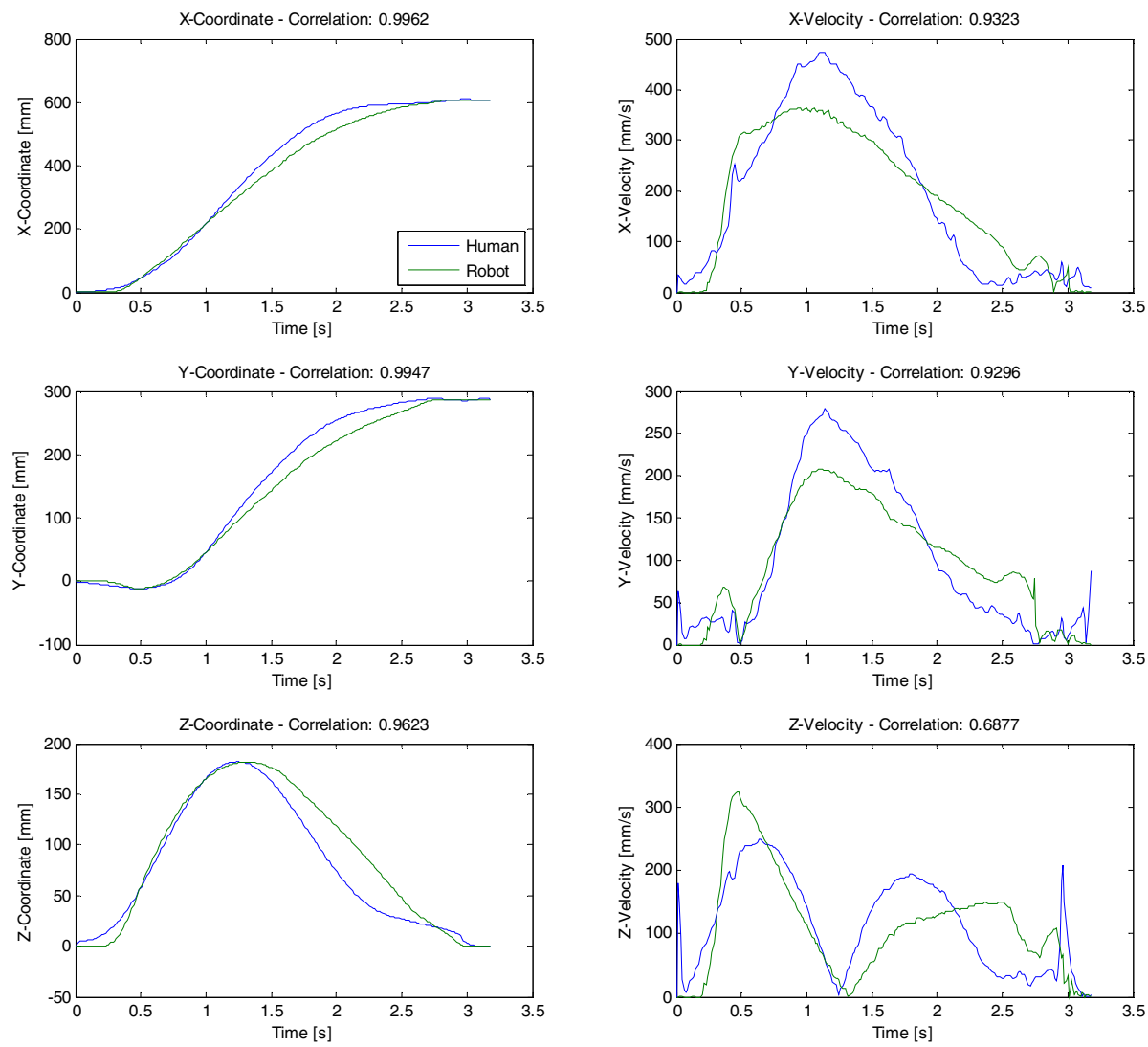

Fig. 4. Exemplary comparison between movement of robot and human 
However, as Figure 5 indicates, this configuration (Max. Acceleration=80\%, Max. Velocity $=20 \%$, Max. Smoothing=20mm - marked red) leads to a time for completion of a movement that is much higher than that of a human, i.e. the robot needs about $250 \%$ of the time that a human would need for that same movement.

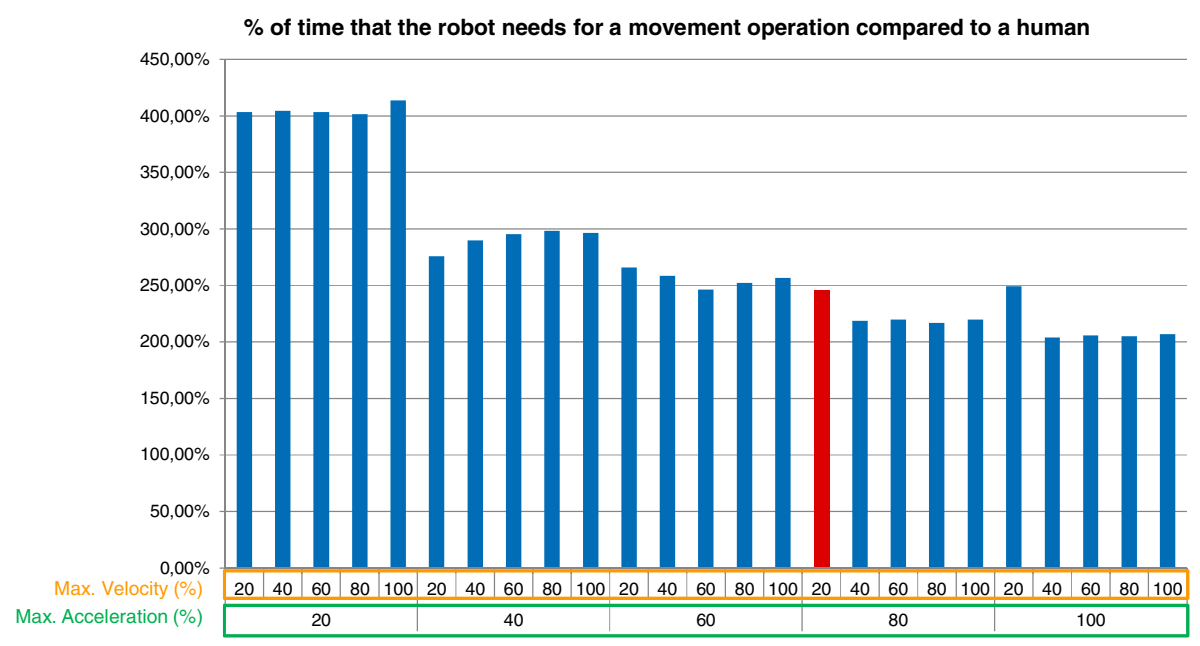

Fig. 5. Comparison between execution times of robot and human for movement to "Row4, Column5" with $20 \mathrm{~mm}$ maximum smoothing

\section{Conclusion}

The presented methodology and techniques allow for the creation of robot movements based on arbitrary human movements. In order to imitate typical human movements in a production system, hand trajectories are obtained by a motion capture system. The recorded positions are extracted and a robot program is generated as well as executed on the respective industrial robot. The exact trajectory of the robot is logged and parameters for the robot motion are adapted in order to optimize that trajectory so that its correlation factor to the human movement increases. After several iterations and the accompanying adaptations, the parameters with the highest correlation between human and robot trajectory - with respect to given restrictions on timespans - are found.

The resulting parameters depend on multiple criteria. First of all the given restrictions in time pose a limit on the velocity, acceleration and smoothing parameter. Additionally the cost function is based on the correlation between robot and human position as well as velocity. For the current evaluation it has been assumed, that these two correlations are of equal importance in regard to the overall movement perception of a human observer. This assumption, however, needs to be verified in empirical studies.

Additionally, the hypothesis of this paper has been that if a robot moves in an anthropomorphic way, this leads to a better anticipation of the robot's trajectory by a 
human observer. In order to validate this hypothesis, the developed framework can be used in empirical studies to test the reaction of human operators for different movements.

Acknowledgements. The authors would like to thank the German Research Foundation DFG for the support of the depicted research within the Research Training Group 1491/2 "Ramp-Up Management: Development of Decision Models for the Production Ramp-Up" and the Cluster of Excellence "Integrative Production Technology for High-Wage Countries".

\section{References}

1. Bainbridge, L.: Ironies of automation. In: Rasmussen, J., Duncan, K., Leplat, J. (eds.) New Technology and Human Error, pp. 775-779. J. Wiley, Chichester (1987)

2. Kinkel, S.: Arbeiten in der Zukunft: Strukturen und Trends der Industriearbeit. Sigma, Berlin (2008)

3. Kuz, S., Heinicke, A., Schwichtenhövel, D., Mayer, M., Schlick, C.: The Effect of Anthropomorphic Movements of Assembly Robots on Human Prediction. In: Proceedings of the 4th International Conference on Applied Human Factors and Ergonomics (AHFE), pp. 1273-1281. USA Publishing, The Printing House, Stoughton (2012)

4. Kupferberg, A., Glasauer, S., Huber, M., Rickert, M., Knoll, A., Brandt, T.: Biological movement increases acceptance of humanoid robots as human partners in motor interaction. Journal of AI \& Society 26(4), 339-345 (2011)

5. Duffy, B.: Anthropomorphism and The Social Robot. Special Issue on Socially Interactive Robots, Robotics and Autonomous Systems 42, 3-4 (2003)

6. Jokisch, D., Troje, N.: Die Wahrnehmung von absoluter Größe in biologischer Bewegung, Ruhr-Universitát-Bochum (2001)

7. Göbel, M.: Verfahren zur intuitiven Programmierung von Industrierobotern durch Demonstration. In: Brecher, C., Klocke, F., Schmitt, R., Schuh, G. (eds.) Berichte aus der Produktionstechnik, Apprimus, Aachen (December 2012) 